\title{
Comparação entre medidas ultra-sônicas e da carcaça na predição da composição corporal em bovinos. Estimativas do peso e da porcentagem dos cortes comerciais do traseiro
}

\author{
Jaime Urdapilleta Tarouco ${ }^{1}$, José Fernando Piva Lobato² ${ }^{2}$ Adriana Kroef Tarouco ${ }^{3}$, Glauco \\ Ivanhoé dos Santos Massia ${ }^{4}$
}

\author{
${ }^{1}$ Zootecnista, bolsista do CNPq. \\ ${ }^{2}$ Depto. de Zootecnia/Fac. Agronomia, UFRGS. Bolsista 1 A CNPq. C.Postal 15.100, CEP: 90001-970, Porto Alegre-RS. \\ ${ }^{3}$ Médico Veterinário, bolsista CNPq. \\ ${ }^{4}$ Médico Veterinário Autônomo.
}

RESUMO - Foram utilizados 102 animais para desenvolver equações de predição a fim de estimar a composição do corte traseiro da carcaça bovina a partir de medidas ultra-sônicas obtidas no animal vivo e comparar estas equações às desenvolvidas a partir de medidas na carcaça. As equações de predição foram desenvolvidas utilizando-se o procedimento estatístico de seleção de variáveis de todas as regressões possíveis e o de seleção de variáveis Stepwise. Os modelos finais utilizando variáveis obtidas no animal vivo incluíram o peso vivo na data do ultra-som (PVUS), a área do músculo Longissimus (AOLUS) e a espessura de gordura subcutânea (EGSUS) para estimar o peso (PCTT; $\mathrm{R}^{2}=0,78$ ) e a porcentagem (RCTT; $\mathrm{R}^{2}=0,18$ ) dos cortes do traseiro. As variáveis obtidas a partir de modelos na carcaça incluíram o peso de carcaça quente (PCQ), a área do músculo Longissimus (AOLC), a espessura de gordura subcutânea (EGSC), o acabamento (ACAB) e a conformação (CONF) para estimação do peso (PCTT; $\left.\mathrm{R}^{2}=0,89\right)$ e da porcentagem (RCTT; $\left.\mathrm{R}^{2}=0,67\right)$ dos cortes do traseiro. As equações de predição desenvolvidas a partir de modelos no animal vivo e na carcaça são úteis para estimar os cortes comerciais do traseiro da carcaça.

Palavras-chave: acabamento, área de olho-de-lombo, bovinos, rendimento de cortes, ultra-som

\section{Comparison between ultrasonic and carcass measures in body composition prediction in cattle. Prediction of weight and percentage hind- quarter retail products}

\begin{abstract}
One hundred two animals were evaluated to develop prediction equations for estimating hind-quarter composition from live animal ultrasound measurements and to compare these equations with those developed from carcass measurements. To develop prediction equations used all-possible regressions procedure for variables reductions and stepwise regression. Final models using live animal variables included live weight (PVUS), Longissimus muscle area (ULMA) and fat thickness (UFAT) for weight (PCTT; $\mathrm{R}^{2}=0.78$ ) and percent $\left(\mathrm{RCTT} ; \mathrm{R}^{2}=0.18\right.$ ) of hind-quarter retail product. The models using variables carcass included hot carcass weight (HCW), carcass Longissimus muscle area (CLMA) and carcass fat thickness (CFAT) and Conformation (CONF) for weight (PCTT; $\left.\mathrm{R}^{2}=0.89\right)$ and percent $\left(\mathrm{RCTT} ; \mathrm{R}^{2}=0.67\right.$ ) of hind-quarter retail product. Results indicate that composition predictions equations developed from live animals and ultrasound measurements can be useful to estimate yield hind-quarter retail product.
\end{abstract}

Key Words: fatness, cattle, Longissimus muscle area, ultrasound, yield retail product

\section{Introdução}

A porção comestível do corte traseiro do animal é uma característica importante para toda a cadeia da carne bovina. No traseiro, localizam-se os cortes comerciais de melhor qualidade e de maior valor comercial da carcaça. Esse corte, no sistema de comercialização brasileiro, representa mais de $50 \%$ do peso da porção comestível da carcaça.
Segundo McKiernan et al. (2005), o rendimento dos cortes desossados constitui um importante componente no desenho de modelos de programas de melhoramento genético para mérito de carcaça de bovinos. Segundo Hamlin et al. (1995), a variação nas características biológicas economicamente importantes é grande e está sob alto grau de controle genético. O efeito do tipo biológico no ritmo relativo de crescimento das características da carcaça avaliadas por ultra-som em diferentes idades e pesos implica 
que a derivação e os ajustes das equações de predição da composição corporal devam ser realizadas especificamente para cada raça ou grupo biológico.

Em carcaças com pesos similares, a porcentagem dos tecidos corporais varia consideravelmente, mas é importante na comercialização. Estudos têm demonstrado que os tecidos muscular e adiposo presentes nos cortes comerciais são parte dos principais critérios considerados pelo consumidor na aquisição do produto (Cuthbertson, 1978).

A técnica de ultra-sonografia tem se mostrado útil para obter informações do rendimento da porção comestível em bovinos vivos (Wallace et al., 1977; Wolcott et al., 2001; Greiner et al., 2003; Tait et al., 2005). As medidas ultrasônicas de sítios anatômicos específicos similares aos observados na carcaça podem contribuir como preditores do peso e da porcentagem de carne e aumentam a exatidão dos modelos de predição no animal vivo diminuindo o tempo necessário para obtenção desta informação em comparação a medidas tradicionais da carcaça.

O criador, estimando o peso e a porcentagem dos cortes comerciais em fase pré-abate, poderia obter vantagens na comercialização de seus animais, pois estaria oferecendo um produto com maior rendimento nos cortes nobres; poderia responder aos sinais de mercado, com alterações no status quo da genética do animal e também modificar suas práticas de manejo, alimentação e comercialização.

O impacto econômico da seleção do rendimento dos cortes comerciais na cadeia da carne pode ser significativo, pois aumenta a disponibilidade da porção comestível dentro de um mesmo peso de carcaça comercializada, elevando seu valor monetário com mesmo custo de produção.

Este trabalho foi realizado com os objetivos de: verificar a acurácia da utilização de medidas obtidas por ultrasom no animal vivo, em relação às medidas realizadas na carcaça, para estimar o peso e a porcentagem da porção comestível do corte traseiro; e desenvolver modelos de predição para o peso e a porcentagem dos cortes comerciais desossados do traseiro.

\section{Material e Métodos}

O estudo foi conduzido em cooperação com os criadores da Conexão Delta G, Dom Pedrito, no Rio Grande do Sul. Foram utilizados 102 animais da raça Braford com diferentes graus de sangue. Os animais apresentavam em média 12 meses de idade e pertenciam a um mesmo grupo contemporâneo e de manejo. Após o desmame, aos seis meses, foram mantidos até o abate confinados e alimentados com ração contendo $16 \%$ de proteína bruta (PB) e $81 \%$ de nutrientes digestíveis totais (NDT). Todos os animais foram identifi- cados com brinco e tatuagem na orelha. Esta identificação foi única, permanente e correlacionada à da carcaça por ocasião do abate, da esfola e da desossa. O peso vivo foi tomado na fazenda, na mesma data da realização das medidas ultra-sônicas (PVUS). Foi considerado o peso do animal individualmente, obtido por meio de balança eletrônica com jejum de sólidos e hídrico de pelo menos 12 horas.

Produção e aquisição das imagens ultra-sônicas (digitalização das imagens) foram obtidas utilizando-se uma unidade principal - eco câmera da marca Aloka SSD 500V (Eletro Medicina Berger, Ltda), equipada com um transdutor linear UST 5049 de 3,5MHz de freqüência e com 17,2 cm de comprimento. As medidas ultra-sônicas da área do músculo Longissimus (AOLUS) e da espessura de gordura subcutânea (EGSUS) foram obtidas entre a $12^{\mathrm{a}}$ e $13^{\mathrm{a}}$ costelas, com auxílio da guia acústica. Após este procedimento, as imagens obtidas foram armazenadas no disco rígido de um computador portátil e interpretadas com o software Animal Ultrasound Service, AUS (AUSKey4W, 1994). Somente uma imagem por animal foi armazenada para cada característica medida por ultra-som. O abate foi realizado em frigorífico comercial, obedecendo ao fluxo normal do estabelecimento. As carcaças foram esfoladas, divididas em meias-carcaças (direita e esquerda), identificadas e armazenadas em câmara de resfriamento para avaliação das medidas rotineiras do Sistema de Tipificação: peso de carcaça quente (PCQ), acabamento (ACAB), maturidade (MAT), sexo (SEX), conformação (CONF). As classificações das características ACAB e CONF foram transformadas em valores numéricos: ACAB; grau $1=0$ a $1 \mathrm{~mm}$; grau 2 = 1 a $3 \mathrm{~mm}$; grau $3=3$ a $6 \mathrm{~mm}$; grau $4=6$ a $10 \mathrm{~mm} \mathrm{e}$ grau 5 = acima de $10 \mathrm{~mm}$; CONF foi utilizada a escala de pontos sugerida por Muller (1980), na qual os algarismos mais elevados correspondem a melhor conformação.

A meia-carcaça direita foi utilizada para a obtenção das medidas de área de olho-de-lombo (AOLC) e espessura de gordura subcutânea (EGSC) e dos pesos e porcentagens dos cortes principais: peso e porcentagem do traseiro (PCT), costela e vazio (PCCV) e dianteiro (PCD). O peso (PCTT) e a porcentagem (RCTT) total da porção comestível do corte do traseiro correspondem à soma dos pesos e percentuais dos cortes individuais comerciais desossados do traseiro, após a realização da toalete das aparas de gordura e do tecido conjuntivo, em relação ao peso do corte traseiro direito (PCT) com osso. As aparas individuais de cada corte foram pesadas após a desossa e, posteriormente, somadas para obtenção do peso das aparas totais do traseiro (PATT). O peso total do osso do corte do traseiro (POSSO) correspondeu à soma total do tecido ósseo do traseiro após a desossa dos cortes do traseiro. As carac- 
terísticas de porcentagem de osso (\%OSSO) e aparas totais (\%ATT) foram obtidas em relação ao peso do corte traseiro com osso (PCT) e multiplicadas por 100. O rendimento de carcaça (REND) foi obtido pela fórmula: PCQ/PVUS $\times 100$.

A espessura de gordura subcutânea (EGSC) utilizada para avaliar a acurácia da medida ultra-sônica foi uma medida não-ajustada, obtida a 3/4 de distância a partir do lado medial do músculo Longissimus para seu lado lateral, aferida com paquímetro. A área de olho-de-lombo (AOLC) foi traçada sobre um papel acetato e posteriormente medida com uma grade plástica de pontuação (AS-235e) distribuída pela Iowa State University of Science and Tecnology, Ames, Iowa, USA (1991).

A análise estatística foi conduzida utilizando-se o SAS (2001). Coeficientes de correlação de Pearson foram utilizados para avaliar as relações entre as medidas no animal vivo, ultra-sônicas e da carcaça e aquelas geradas a partir da relação destas medidas.

As equações de predição foram desenvolvidas pelo procedimento de construção do modelo utilizando-se o Método de Seleção de Variáveis de todas as Regressões Possíveis e pelo método de seleção de variáveis para decidir a inclusão e permanência destas variáveis nos modelos. No método de seleção de variáveis Stepwise, considerou-se nível de significância de $20 \%$ para inclusão de variáveis e de $15 \%$ para a permanência no modelo.

As equações de predição desenvolvidas pelo procedimento de seleção de variáveis de todas as regressões possíveis incluíram variáveis a partir de medidas obtidas no animal vivo e a partir das características de carcaça como variáveis independentes. As medidas de carcaça utilizadas para desenvolver as equações de predição foram: peso de carcaça quente (PCQ); acabamento (ACAB); conformação (CONF); área do músculo Longissimus (AOLC); espessura de gordura subcutânea na 12aㅡ costela (EGSC); e como covariáveis a porcentagem do osso do traseiro (\%OSSO) e a porcentagem das aparas totais do corte traseiro (\%ATT). As equações foram avaliadas em relação ao coeficiente de determinação ( $\mathrm{R}^{2}$ ) e ao erro-padrão de predição (RMSE) com o Cp descrito por Mallow (1973), citado por MacNeil (1983). Para modelos com um ajuste fino, o Cp se aproxima ao número de variáveis preditoras (MacNeil, 1983). Efeitos genéticos não foram considerados no processo de modelagem.

\section{Resultados e Discussão}

As médias das características obtidas no animal vivo (Tabela 1) foram 297,77 kg com amplitude de $104 \mathrm{~kg}$; $52,68 \mathrm{~cm}^{2}$ com amplitude de $26,21 \mathrm{~cm}^{2}$ e de $3,94 \mathrm{~mm}$ com amplitude de $6 \mathrm{~mm}$ para o peso vivo na data do ultra-som, a área de olho-de-lombo e a espessura de gordura subcutânea, respectivamente.

A média para o conjunto de dados para o RCTT foi de 68,53\%, com variação de 8,31\%. O resultado do RCTT deste estudo está na amplitude de variação de 36,37 a 76,2\%, obtidas por diversos autores na literatura (Crouse \& Dikeman, 1976; Herring et al., 1994; Hamlin et al., 1995a; Williams et al., 1997; May et al., 2000; Realini et al., 2001; Greiner et al., 2003; Tait et al., 2005). O desvio-padrão do RCTT de 1,67\% foi semelhante ao obtido por Herring et al. (1994), 1,60\%. Entretanto, resultados superiores foram obtidos por Crouse \& Dikeman (1976), 3,59\%; Hamlin et al. (1995a), 4,10\%; Williams et al. (1997), 3,30\%; May et al. (2000), 2,94\%; Realini et al. (2001), 2,5\%; Greiner et al. (2003), 4,2\%; e Tait et al. (2005), 2,22\%, o que está relacionado à variação fenotípica dos tipos biológicos dos animais, à porcentagem de gordura na carcaça, aos sistemas de toaletes adotados nas carcaças e aos cortes utilizados pelos autores em suas amostragens. A magnitude da variação fenotípica desta característica depende altamente da composição genética dos indivíduos, do manejo nutricional prévio, do sexo, da idade e do peso na data das avaliações.

As médias das características de carcaça PCQ, EGSC, ACAB, CONF, AOLC, PATT e \%ATT para o conjunto de dados para o desenvolvimento dos modelos foram de $158,25 \mathrm{~kg} ; 3,73 \mathrm{~mm} ; 3,04 ; 7,43 ; 52,53 \mathrm{~cm}^{2} ; 4,40$ kg e $10,82 \%$, respectivamente.

Tanto o peso vivo como as características de carcaça foram as variáveis mais intensamente correlacionadas ao PCTT (Tabela 2), com coeficientes de correlação simples de 0,85 a 0,93 para o PVUS e PCQ, respectivamente. Utilizando diferentes procedimentos de corte para tamanho, localização, grau de acabamento das carcaças, raça e peso de abate, outros autores (Perkins, 1992; Herring et al., 1994; Williams et al., 1997; Realini et al., 2001; Greiner et al., 2003) obtiveram resultados semelhantes, de modo que o peso vivo foi a variável mais estreitamente correlacionada ao peso da porção comestível da carcaça, com coeficientes de correlação linear simples de 0,77 a 0,91. Resultados similares foram obtidos por Abraham et al. (1968), $r=0,95$; Busch et al. (1968), $r=0,89$; Cross et al. (1973), $r=0,88$; Williams et al. (1997), $r=0,90$; Realini et al. (2001), $r=0,90$; Greiner et al. (2003), $r=0,83$, entre o peso de carcaça e o peso da porção comestível da carcaça.

A variável POSSO apresentou relação também alta, de 0,73, com o PCTT, provavelmente em decorrência da importante proporção deste tecido no peso do corte traseiro, de 20,76\% do PCT e 30,22\% do PCTT, com variação de 4,54 kg, que corresponde uma amplitude percentual de 7,88\%. Esse resultado também pode ser explicado pela afirmação de 
Tabela 1 - Características do animal vivo e na carcaça para o conjunto de animais utilizado no desenvolvimento do modelo

Table 1 - Traits of live animal and in carcass for set of animals used in development of the model

\begin{tabular}{lrrrr}
\hline $\begin{array}{l}\text { Característica } \\
\text { Trait }\end{array}$ & $\begin{array}{c}\text { Média } \\
\text { Mean }\end{array}$ & $\begin{array}{c}\text { DPM } \\
\text { SD }\end{array}$ & $\begin{array}{r}\text { Mínimo } \\
\text { Minimum }\end{array}$ & $\begin{array}{r}\text { Máximo } \\
\text { Maximum }\end{array}$ \\
\hline PVUS (FUW) & 297,77 & 9,39 & 245,00 & 379,00 \\
AOLUS (ULMA) & 52,68 & 5,29 & 41,29 & 67,50 \\
EGSUS (UFAT) & 3,94 & 1,23 & 1,80 & 7,80 \\
PCQ (HCW) & 158,25 & 16,28 & 130,20 & 218,00 \\
ACAB (FAT) & 3,04 & 0,66 & 2,00 & 4,00 \\
CONF (CONF) & 7,43 & 1,13 & 4,00 & 9,00 \\
AOLC (CLMA) & 52,53 & 5,51 & 40,63 & 66,43 \\
EGSC (CFAT) & 3,73 & 1,35 & 1,50 & 8,00 \\
PCT (WHQ) & 37,74 & 3,73 & 31,50 & 52,00 \\
PCTT (WRPH) & 25,87 & 2,68 & 20,85 & 36,45 \\
RCTT (RRPH) & 68,53 & 1,67 & 65,14 & 73,45 \\
POSSO (WBH) & 7,82 & 0,78 & 6,26 & 10,80 \\
\%OSSO (\%BH) & 20,76 & 1,32 & 17,98 & 25,86 \\
PATT (WFTRIM) & 4,40 & 0,81 & 2,86 & 6,32 \\
\%ATT (\%FTRIM) & 10,82 & 1,58 & 7,48 & 14,58 \\
\hline
\end{tabular}

${ }^{1} \mathrm{PVUS}=$ peso vivo na data do ultra-som ( $F W T$ = final unshrunk weight); AOLUS = área de olho-de-lombo por ultra-som (ULMA = ultrasound Longissimus muscle area); EGSUS = espessura de gordura por ultra-som (UFAT = ultrasound fat thickness); $\mathrm{PCQ}=$ peso de carcaça quente $(\mathrm{HCW}=$ hot carcass weight $) ; \mathrm{ACAB}=$ acabamento ( $F A T=$ visual fat score) $; \mathrm{CONF}=$ conformação $(C O N F=$ conformation $) ; \mathrm{AOLC}=$ área de olho-de-lombo na carcaça (CLMA = carcass Longissimus muscle area); EGSC = espessura de gordura na carcaça (CFAT = carcass thickness fat); $\mathrm{PCT}=\%$ de traseiro $(W H Q=$ percent hindquarter $)$; $\mathrm{PCTT}=$ peso da porção comestível do corte traseiro (WRPH = weight of hindquarter retail product); RCTT = porcentagem da porção comestível do corte traseiro $(R R P H=$ percent of hindquarter retail product); POSSO = peso total do osso do corte traseiro $(\mathrm{WBH}=$ weigth of hindquarter bone); \%OSSO $=\%$ do osso do corte traseiro ( $\% \mathrm{BH}=$ percent of hindquarter bone); PATT $=\%$ aparas totais do corte traseiro (WFTRIM = weigth of hindquarter fat trim); $\%$ ATT $=\%$ aparas totais do corte traseiro (\%FTRIM = percent of hindquarter fat trim).

Nathusius, citado por Hammond (1932), de que os tecidos originados da mesma camada embrionária, mesoderma, estão correlacionados ao seu desenvolvimento embrionário. Conseqüentemente, o crescimento do músculo e do osso deveria estar positivamente relacionado.

Conforme Hammond (1932), o tamanho do osso e sua forma (dimensões) são os fatores determinantes do tamanho e da forma do músculo, o que está de acordo com a sugestão de Stewart (1972) de que o desenvolvimento do osso e o peso do músculo podem não ser independentes.

Assim, o desenvolvimento do músculo segue o desenvolvimento do osso. Estas conclusões foram confirmadas pelo estudo de Shahin \& Berg (1987), em que os coeficientes de crescimento dos grupos de músculos, com exceção da parte distal dos membros, indicaram que os músculos dos animais da raça Hereford aumentaram em peso proporcionalmente ao osso.

O PVUS não foi correlacionado significativamente $(r=-0,06, P=0,5299)$ com o RCTT, o que está de acordo com os resultados de Wallace et al. (1977), Williams et al. (1997), May et al. (2000) e Realini et al. (2001), que reportaram que o peso vivo não é uma variável correlacionada significati-
Tabela 2 - Correlações simples das características com o peso (PCTT) e a porcentagem (RCTT) da porção comestível do corte traseiro

Table 2 - $\quad$ Simple correlations of traits with weight (WRPH) and retail product hindquarter percent (RRPH)

\begin{tabular}{lcccc}
\hline $\begin{array}{l}\text { Característica } \\
\text { Trait }\end{array}$ & $\begin{array}{c}\text { RCTT (\%) } \\
\text { RRPH }\end{array}$ & $\begin{array}{c}\text { Valor P } \\
\text { P-value }\end{array}$ & $\begin{array}{c}\text { PCTT (kg) } \\
\text { WRPH }\end{array}$ & $\begin{array}{c}\text { Valor P } \\
\text { P-value }\end{array}$ \\
\hline PVUS & $-0,06$ & $\mathrm{P}=0,5299$ & 0,85 & $\mathrm{P}<0,0001$ \\
AOLUS & 0,33 & $\mathrm{P}<0,0006$ & 0,55 & $\mathrm{P}<0,0001$ \\
EGSUS & 0,02 & $\mathrm{P}<0,8680$ & 0,32 & $\mathrm{P}<0,0010$ \\
PCQ & 0,03 & $\mathrm{P}<0,7756$ & 0,93 & $\mathrm{P}<0,0001$ \\
AOLC & 0,35 & $\mathrm{P}<0,0003$ & 0,50 & $\mathrm{P}<0,0001$ \\
EGSC & 0,05 & $\mathrm{P}<0,6174$ & 0,31 & $\mathrm{P}<0,0015$ \\
ACAB & 0,07 & $\mathrm{P}<0,4836$ & 0,29 & $\mathrm{P}<0,0032$ \\
CONF & $-0,26$ & $\mathrm{P}<0,0093$ & 0,07 & $\mathrm{P}<0,5005$ \\
\%OSSO & $-0,36$ & $\mathrm{P}<0,0002$ & - & - \\
\%ATT & $-0,65$ & $\mathrm{P}<0,0001$ & - & - \\
POSSO & - & - & 0,73 & $\mathrm{P}<0,0001$ \\
PATT & - & - & 0,31 & $\mathrm{P}<0,0014$ \\
\hline
\end{tabular}

1 Ver Tabela 1 para descrição dos acrônimos.

${ }^{1}$ See Table 1 for description of acronyms.

vamente $(\mathrm{r}=0,01, \mathrm{P}>0,05 ; \mathrm{r}=-0,22, \mathrm{P}>0,05 ;(\mathrm{r}=0,02, \mathrm{P}>0,05$; e $r=-20, P>0,05$, respectivamente) com a porcentagem de cortes comerciais da carcaça. O resultado obtido neste experimento pode ser atribuído à pequena porcentagem da porção comestível do traseiro $(8,70 \%)$ em relação ao peso vivo dos animais e à pequena variação nesta característica $(\mathrm{DP}=2,68 \mathrm{~kg})$. O efeito da raça sobre esta relação pode estar presente, principalmente, quando o peso vivo é medido na data do abate (Wolcott et al., 2001). Em raças com tendência de apresentar ao abate um grau de acabamento mais elevado, a influência negativa do peso vivo sobre o rendimento da porção comestível da carcaça é maior, em razão da elevada porcentagem de gordura de recorte nos cortes comerciais.

O coeficiente de correlação do PVUS com o RCTT apresentou sinal negativo, o que está de acordo com resultado relatado por Wolcott et al. (2001) de que o peso vivo aumenta enquanto o RCTT diminui. Esse resultado pode explicar a magnitude dos coeficientes de correlação mais elevados obitdos por esses autores em relação aos deste estudo.

A relação entre AOLUS com o PCTT neste estudo foi de $0,55(\mathrm{P}=0,0001)$. Crouse et al. (1975) indicaram que a medida da área do músculo pode ser útil para explicar a variação na quantidade de carne na carcaça. Cross et al. (1973) afirmaram que a área de músculo pode ser mais preditiva em determinados grupos de carcaças que apresentem uma pequena variabilidade no grau de acabamento, enquanto May et al. (2000) concluíram que a variável AOLUS é significativa em equações desenvolvidas, com medidas no animal vivo, para predição do peso dos cortes comerciais. 
A AOLUS ( $\mathrm{r}=0,33, \mathrm{P}=0,0006)$ é a variável, das obtidas no animal vivo, mais intensamente correlacionada com a RCTT. Resultados similares em relação à magnitude das relações entre a AOLUS e a porcentagem de cortes comerciais da carcaça, de $r=0,40$ e $r=0,30$, foram obtidos por Realini et al. (2001) e Tait et al. (2005); respectivamente. Coeficiente de correlação mais baixo, porém significativo $(r=0,17, P<0,10)$, foi obtido Greiner et al. (2003). As discrepâncias nos resultados dos diferentes trabalhos podem ser atribuídas, em parte, à utilização de covariáveis nos modelos, assim como, nenhum só divisor pode remover totalmente o efeito do peso vivo ou de carcaça na característica AOLUS.

Segundo Wolcott et al. (2001), a AOLUS tem grande impacto na estimativa do rendimento dos cortes comerciais em animais que não tenham ainda depositado suficiente gordura, obtendo aumento na área de lombo, com a correspondente elevação no RCTT. Isto, de certa forma, ocorreu neste experimento, pois os animais eram jovens e não apresentavam grau de acabamento elevado, de modo que a maior parte da gordura subcutânea participou positivamente no peso do corte.

Resultados similares para AOLC e PCTT foram obtidos por Cole et al. (1960), que encontraram coeficientes de correlação simples entre a AOLC e o peso de carne magra total da carcaça de 0,43 e de 0,40 a 0,55 com a quantidade de carne magra em diferentes cortes da carcaça. Greiner et al. (2003) e Tait et al. (2005) obtiveram coeficientes de correlação entre a AOLC e a porcentagem de carne nos quatro cortes primários iguais a 0,31 e 0,33.

Abraham et al. (1968) obtiveram coeficiente de correlação similar ao ( $r=0,32$; Tabela 2 ) entre EGSC o peso dos cortes comerciais desossados da carcaça igual a $r=0,25$. O coeficiente de correlação entre a EGSUS e RCTT foi de $r=0,02$ $(\mathrm{P}=0,8680)$. Associações médias entre estas características foram obtidas por Herring et al. (1994), $r=-0,42, P<0,01$, Williams et al. (1997), $r=-0,38, \mathrm{P}<0,01$; e Realini et al. (2001), $\mathrm{r}=-0,33, \mathrm{P}<0,10$.

Na Tabela 3 constam os modelos completos de predição para PCTT e a RCCT a partir de medidas obtidas no animal vivo. No desenvolvimento do modelo utilizando características obtidas no animal vivo para predição do PCTT, verificou-se que o modelo deveria incluir pelo menos as variáveis PVUS $(\mathrm{P}<0,001)$ e AOLUS $(\mathrm{P}<0,001)$. O PVUS $(r=0,85, P<0,001)$ é a variável mais intensamente relacionada ao PCTT, portanto, a primeira variável a entrar em um procedimento de seleção de variáveis por Stepwise.

Na construção do modelo utilizando-se um procedimento de seleção de todas as regressões possíveis (Tabela 3) com base no $\mathrm{R}^{2}$, a maior parte da variação do PCTT foi explicada pelo PVUS (73\%), considerando o ajuste só com o PVUS. O modelo deveria incluir pelo menos as variáveis PVUS e AOLUS. Esse resultado era esperado, uma vez que houve mais alta correlação destas variáveis e, segundo Herring et al. (1994), o peso vivo está diretamente relacionado ao tamanho e ao peso dos cortes comerciais.

Com inclusão da EGSUS, tem-se uma explicação de 78\% e um Cp considerado ideal de 4 . Geralmente, pesquisadores têm reportado que a espessura de gordura subcutânea obtida por ultra-som participa da equação com sinal negativo, ou seja, quanto maior quantidade de gordura menor o peso dos cortes comerciais (Wallace et al., 1977; Herring et al., 1994; Greiner et al., 2003). Entretanto, neste estudo a EGSUS foi positivamente correlacionada ao PCTT $(r=0,32$, $P=0,0010$ ), provavelmente em razão do baixo grau de acabamento das carcaças, sugerindo que grande parte da gordura estava presente no peso dos cortes e também que houve pouco recorte deste tecido na toalete da carcaça. Isso pode ser confirmado pela porcentagem de aparas totais no corte traseiro, que foi em média de 10,8\% neste conjunto de animais. Esses resultados demonstram que quando o grau de acabamento é baixo, menos de $5 \mathrm{~mm}$, a porcentagem de aparas totais também é pequena, pois este tecido faz parte do peso da porção comestível e influencia positivamente na predição desta característica.

A maior parte da variação do RCTT é explicada pela AOLUS (11\%, Tabela 3), quando se considera no ajuste somente a área do músculo Longissimus. Entretanto, este modelo apresentou um viés alto, Cp de Mallow de 8 e um erro padrão de predição (RMSE) de 1,59\%. Hamlin et al. (1995b) obtiveram resultados semelhantes ao deste estudo, de modo que a área do músculo explicou menos que 15\% a porcentagem da porção comestível dos cortes da carcaça.

A inclusão do PVUS no modelo elevou a explicação para 16\%, com a redução no valor do Cp para 3,99 e do RMSE para $1,55 \%$. Este pequeno acréscimo no coeficiente de determinação com a inclusão do PVUS pode ser atribuído ao fato de que a relação entre o peso vivo e o rendimento total de cortes comerciais decresce à medida que se fraciona a quantidade total de carne em pequenos cortes da carcaça (Herring et al., 1994). A adição de cortes menores para se chegar ao peso total introduz o viés da desossa e do recorte de gordura, sendo mais pronunciado quanto mais subdivisões se fizer na carcaça. O peso vivo está mais correlacionado ao peso dos cortes comerciais que à porcentagem dos cortes na carcaça (Wallace et al, 1977; Williams et al., 1997; Realini et al., 2001; Tait et al., 2005).

Com a inclusão da EGSUS, tem-se 18\% de explicação no RCTT, um Cp de Mallow de 4 e um erro-padrão de predição (RMSE) de 1,54\%. A justificativa para a inclusão da variável 
Tabela 3 - Modelos de predição para o peso (PCTT) e a porcentagem (RCTT) da porção comestível do corte traseiro a partir de medidas obtidas no animal vivo

Table 3 - Prediction of models for retail product hindquarter weight $(W R P H)$ and percent (RRPH) from live animal measures

\begin{tabular}{|c|c|c|c|c|c|c|c|}
\hline Item $^{1}$ & $\mathrm{R}^{2}$ & $\begin{array}{l}\text { Cp } \\
C p\end{array}$ & $\begin{array}{l}\text { MSE } \\
\text { MSE }\end{array}$ & $\begin{array}{l}\text { RMSE } \\
\text { RMSE }\end{array}$ & \multicolumn{3}{|c|}{$\begin{array}{c}\text { Variável no modelo }{ }^{1} \\
\text { Variable in model }\end{array}$} \\
\hline \multicolumn{8}{|c|}{ PCTT (WRPH) } \\
\hline & 0,73 & 20,68 & 1,97 & 1,40 & & $\begin{array}{l}\text { PVUS } \\
F W T\end{array}$ & \\
\hline & 0,77 & 3,75 & 1,67 & 1,29 & & $\begin{array}{l}\text { AOLUS } \\
\text { ULMA }\end{array}$ & $\begin{array}{l}\text { PVUS } \\
F W T\end{array}$ \\
\hline & 0,78 & 4,00 & 1,66 & 1,29 & $\begin{array}{l}\text { EGSUS } \\
\text { AOLUS }\end{array}$ & $\begin{array}{l}\text { PVUS } \\
\text { UFAT }\end{array}$ & $\begin{array}{l}\text { ULMA } \\
F W T\end{array}$ \\
\hline
\end{tabular}

RCTT (RRPH)

\begin{tabular}{|c|c|c|c|c|c|c|}
\hline 0,11 & 8,18 & 2,52 & 1,59 & & $\begin{array}{c}\text { AOLUS } \\
\text { ULMA }\end{array}$ & \\
\hline 0,16 & 3,99 & 2,40 & 1,55 & & AOLUS & PVUS \\
\hline 0,18 & 4,00 & 2,37 & 1,54 & EGSUS & $\begin{array}{l}\text { ULMA } \\
\text { PVUS }\end{array}$ & $\begin{array}{c}F W T \\
\text { ULMA }\end{array}$ \\
\hline
\end{tabular}

${ }^{1}$ Ver Tabela 1 para descrição dos acrônimos.

${ }^{1}$ See Table 1 for description of acronyms.

EGSUS se deve à facilidade de obtenção desta medida, ao valor do Cp $(4,000)$ considerado ideal e à diminuição do RMSE para $1,54 \%$.

O PCTT a partir de medidas obtidas no animal vivo foi predito com boa magnitude se considerados os coeficientes de determinação nas equações 1 e 2 (Tabela 4). As medidas AOLUS e EGSUS, obtidas por ultra-som, quando incluídas no modelo, aumentaram o poder de explicação e diminuíram o viés e erro de predição do modelo. Resultados similares ao da equação 1 foram obtidos por Herring et al. (1994), que encontraram coeficiente de determinação de 0,76 utilizando as mesmas características para estimar o peso dos cortes comerciais da carcaça.

Resultado similar ao deste trabalho foi obtido por Realini et al. (2001), que reportaram $\mathrm{R}^{2}$ de 0,80 utilizando as mesmas variáveis da equação 2 para estimar o peso dos cortes comerciais da carcaça. Coeficientes de determinação mais elevados foram obtidos por Williams et al. (1997) e Greiner et al. (2003), de 0,84 e 0,83, respectivamente, para as variáveis da equação 2 deste estudo na estimativa dos pesos dos cortes comerciais da carcaça.

As equações 3 e 4 (Tabela 4) comprovam, pelos coeficientes de determinação de 0,16 e 0,18 , que a porcentagem da porção comestível do corte traseiro da carcaça não foi altamente explicada pelas variáveis obtidas no animal vivo.

Resultados semelhantes ao deste estudo (equação 2) foram obtidos por Williams et al. (1997), que relataram $\mathrm{R}^{2}$ de 0,17 na explicação da porcentagem de cortes comerciais da carcaça. Realini et al. (2001) relataram valor do $\mathrm{R}^{2}$ de 0,37 na explicação da porcentagem de cortes comerciais da carcaça utilizando 32 novilhos Hereford. Resultados mais elevados foram obtidos por May et al. (2000), que encontraram $\mathrm{R}^{2}$ de 0,57 , e por Tait et al. (2003), que obtiveram coeficiente de determinação de 0,74 na explicação da porcentagem de carne magra no corte traseiro. Greiner et al. (2003) obtiveram $R^{2}$ de 0,57 na explicação da porcentagem dos cortes comerciais desossados da carcaça. Tait et al. (2000) relataram que as características, como as utilizadas na equação 4 deste estudo, explicaram $42 \%$ da porcentagem dos cortes comerciais da carcaça.

A diferença dos resultados deste experimento pode estar relacionada ao tamanho da amostra, a maior variabilidade na composição corporal e à utilização de animais de diferentes grupos biológicos; ao peso e ao nível nutricional. Essas variações influenciam o grau de acabamento e o recorte da gordura. Outro fator seria a utilização de todos os cortes comerciais da carcaça aumentando a proporção das partes em relação ao todo do animal. Greiner et al. (2003) obtiveram o valor do erro-padrão de predição mais elevado (RMSE $=2,79 \%$ ) utilizando as mesmas variáveis no modelo completo a partir de características obtidas no animal vivo. No entanto, Tait et al. (2005) encontraram valor do erro-padrão de predição de 1,62\% semelhante ao deste estudo (1,54\%), apesar de incluírem uma variável a mais, a área do músculo Gluteus medius.

Segundo Kempster et al. (1982), o coeficiente de correlação é influenciado pela amplitude de valores da amostra em que é baseado. Apesar de satisfatório para comparar preditores na mesma amostra de carcaças, não é apropriado para comparação da precisão obtida em diferentes amostras. Quando essas comparações são realizadas, o erro-padrão de predição é o melhor critério, pois, além de estar relacionado ao coeficiente de correlação, considera a variação na variável dependente na explicação. Essas considerações são importantes, pois apesar de os coeficientes de determinação $\left(\mathrm{R}^{2}\right)$ encontrados terem sido de baixa magnitude, os valores dos erros-padrão de predição (RMSE) foram baixos (<2\%) para o modelo de estimação a partir de características obtidas no animal vivo dentro de um mesmo grupo contemporâneo.

Os resultados obtidos neste estudo para os modelos de predição do PCTT (Tabela 5) corroboram os descritos por Realini et al. (2001) e Greiner et al. (2003). Segundo esses autores, apesar de os modelos de predição utilizando características obtidas no animal vivo explicarem grande parte da variação do peso da porção comestível da carcaça, esses modelos utilizam características de carcaça com $\mathrm{R}^{2}$ maior. 
Tabela 4 - Equações de predição para estimar o peso (PCTT) e a porcentagem (RCTT) da porção comestível do corte do traseiro a partir de medidas in vivo

Table 4 - $\quad$ Prediction equations to estimate for retail product hindquarter weight (WRPH) and percent (RRPH) from live measures

\begin{tabular}{|c|c|c|c|c|c|c|c|}
\hline \multirow[b]{2}{*}{$\begin{array}{l}\text { Variável dependente e equação }{ }^{1} \\
\text { Dependent variable and equation }\end{array}$} & \multirow[b]{2}{*}{$\mathrm{R}^{2}$} & \multirow[b]{2}{*}{$\begin{array}{l}\text { RMSE } \\
\text { RMSE }\end{array}$} & \multirow[b]{2}{*}{$\begin{array}{l}\mathrm{Cp} \\
\mathrm{Cp}\end{array}$} & \multicolumn{4}{|c|}{$\begin{array}{l}\text { Coeficiente de regressão parcial } \\
\text { Coefficient of partial regression }\end{array}$} \\
\hline & & & & $\begin{array}{l}\text { Intercepto } \\
\text { Intercept }\end{array}$ & $\begin{array}{c}\text { EGSUS }(\mathrm{mm}) \\
\text { UFAT }\end{array}$ & $\begin{array}{c}\text { AOLUS }\left(\mathrm{cm}^{2}\right) \\
\text { ULMA }\end{array}$ & $\begin{array}{l}\text { PVUS (kg) } \\
\quad F W T\end{array}$ \\
\hline \multicolumn{8}{|l|}{ PCTT (WRPH) } \\
\hline 1 & 0,77 & 1,29 & 3,75 & $-0,80$ & - & 0,12 & 0,07 \\
\hline \multicolumn{8}{|l|}{ RCTT (RRPH) } \\
\hline 3 & 0,16 & 1,55 & 3,99 & 65,46 & - & 0,14 & $-0,01$ \\
\hline 4 & 0,18 & 1,54 & 4,00 & 65,27 & 0,19 & 0,15 & $-0,02$ \\
\hline
\end{tabular}

1 Ver Tabela 1 para descrição dos acrônimos.

1 See Table 1 for description of acronyms.

Tabela 5 - Modelos de predição para o peso (PCTT) e a porcentagem (RCTT) da porção comestível do corte traseiro a partir de medidas obtidas na carcaça

Table 5 - $\quad$ Predictions models for retail product hindquarter weight (WRPH) and percent (RCTT) from carcass measures

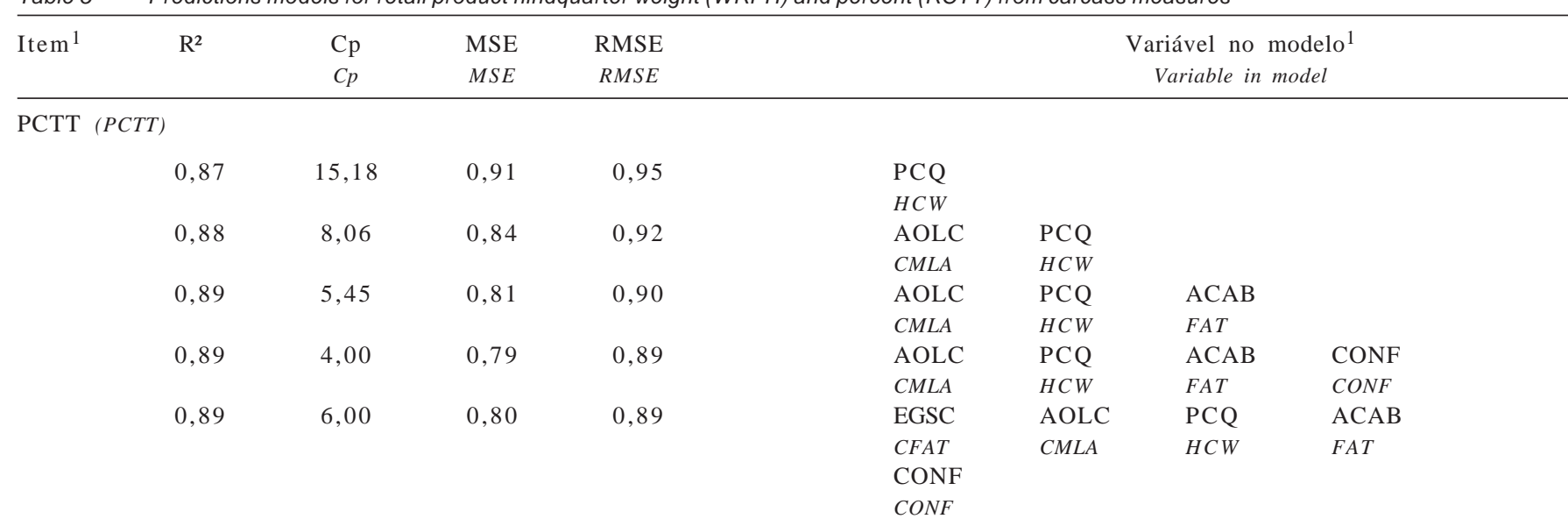

RCTT (RCTT)

\begin{tabular}{|c|c|c|c|c|c|c|c|c|}
\hline 0,57 & 25,20 & 1,22 & 1,10 & $\begin{array}{l}\% \text { OSSO } \\
\% \text { BONE }\end{array}$ & $\begin{array}{l}\% \mathrm{ATT} \\
\% \text { FTRIM }\end{array}$ & & & \\
\hline 0,63 & 10,72 & 1,06 & 1,03 & $\begin{array}{l}\text { AOLC } \\
C M L A\end{array}$ & & & & \\
\hline 0,66 & 4,28 & 0,99 & 0,99 & $\begin{array}{l}\text { AOLC } \\
C M L A\end{array}$ & $\begin{array}{l}\text { PCQ } \\
H C W\end{array}$ & & & \\
\hline 0,67 & 4,92 & 0,98 & 0,99 & $\begin{array}{l}\text { AOLC } \\
C M L A\end{array}$ & $\begin{array}{l}\text { PCQ } \\
H C W\end{array}$ & $\begin{array}{l}\text { CONF } \\
\text { CONF }\end{array}$ & & \\
\hline 0,67 & 8,00 & 0,99 & 0,99 & $\begin{array}{l}\text { EGSC } \\
C F A T\end{array}$ & $\begin{array}{l}\text { AOLC } \\
C M L A\end{array}$ & $\begin{array}{l}\text { PCQ } \\
H C W\end{array}$ & $\begin{array}{l}\text { ACAB } \\
F A T\end{array}$ & $\begin{array}{l}\text { CONF } \\
\text { CONF }\end{array}$ \\
\hline
\end{tabular}

1 Ver Tabela 1 para descrição dos acrônimos.

1 See Table 1 for description of acronyms.

Esses altos valores do coeficiente de determinação, reportados na predição do PCTT usando variáveis da carcaça, comparados aos obtidos no animal vivo, são, em grande parte, atribuídos à diferença na variação inicial explicada pelo peso vivo versus o peso de carcaça quente. O peso de carcaça quente explicou sozinho $14,62 \%$ a mais da variação inicial do PCTT em comparação ao peso vivo.
Portanto, diferenças no rendimento de carcaça, ou seja, relação peso carcaça/peso vivo, podem explicar grande parte das diferenças dos valores dos coeficientes de determinação entre as equações a partir de medidas no animal vivo e na carcaça obtidas para a predição do peso da porção comestível da carcaça (Greiner et al., 2003). 
Verificou-se, pela análise realizada, que o modelo deveria incluir pelo menos as variáveis PCQ, AOLC e ACAB e que as covariáveis POSSO e PATT não são importantes.

Na construção do modelo para as características de carcaça utilizando-se o procedimento de seleção de variáveis de todas as regressões possíveis com base no $R^{2}$, a maior parte da variação do PCTT foi explicada pelo PCQ (87,5\%) considerando o ajuste somente com o PCQ, resultado já esperado se considerada a mais alta correlação destas variáveis. No entanto, este modelo tem um viés alto, um Cp de Mallow de 15 (Tabela 5) não indicado.

A inclusão da AOLC no modelo elevou a explicação para 88,5\%, com a redução do Cp para 8 . O menor impacto na elevação do coeficiente de determinação com a inclusão da variável AOLC (1\%) em relação à AOLUS (4\%) no modelo, pode ser explicada, em parte, pela menor correlação da área do músculo Longissimus obtida na carcaça $(\mathrm{r}=0,50, \mathrm{P}<0,0001)$ em relação à apresentada pela AOLUS ( $r=0,55, P<0,0001)$ com o peso da porção comestível do corte traseiro. Essa menor relação pode estar relacionada a fatores ligados a acurácia na obtenção das medidas. Investigações de modelos com três variáveis seriam interessantes. Os que incluem a AOLC, PCQ e ACAB, pelo valor do $C p$ obtido e aquele que inclui a EGSC, AOLC, PCQ, pelo fato de conter uma medida objetiva da espessura de gordura subcutânea que está inserida nos sistemas de avaliação de carcaças e ser determinante no preço a ser pago pela carcaça no sistema de comercialização.

As variáveis ACAB e EGSC se correlacionaram entre si $(\mathrm{r}=0,60, \mathrm{P}<0,0001)$ e foram medianamente correlacionadas ao PCTT, $r=0,29(P=0,0032)$ e $r=0,31(P=0,0015)$, respectivamente. A inclusão destas medidas no modelo diminuiu o viés de predição do PCTT e, apesar de não estarem estreitamente correlacionadas à variável dependente, neste caso, diminuiu o erro-padrão de predição do modelo (RMSE $=0,90)$ em relação ao modelo com as variáveis PCQ e AOLC (RMSE = 0,92).

Utilizando o procedimento de seleção de variáveis Stepwise, o modelo selecionado foi composto de quatro variáveis preditoras (PCQ, AOLC, ACAB, e CONF, nesta ordem de inclusão). A inclusão da conformação deveu-se à diminuição do viés da predição $(\mathrm{Cp}=4,00)$ e foi selecionada para participar do modelo pelo procedimento Stepwise. Segundo Neter et al. (1990), em algumas situações, é desejável considerar mais de um critério para avaliação de possíveis variáveis preditoras para inclusão no modelo.

Os resultados de seleção de variáveis, tanto considerando todas as regressões possíveis como no procedimento Stepwise, não se alteraram com a inclusão das covariáveis, o que está relacionado ao fato de que POSSO e PATT são correlacionadas a PCQ. Previa-se que a inclusão desta característica no modelo eliminasse a influência das covariáveis.

As covariáveis porcentagem de osso e porcentagem de aparas totais explicaram 57\% da variação da RCTT, com um Cp de 25 e um RMSE de 1,10\% (Tabela 5). A inclusão destas covariáveis no modelo é importante porque representam porção significativa do peso do traseiro, perfazendo 31,21\% do seu peso. Além disso, essas covariáveis estão correlacionadas significativamente e negativamente à porcentagem da porção comestível (Tabela 3). Segundo Kempster et al. (1977), a adição do peso e ou da porcentagem dos ossos do corte melhora a estabilidade e a precisão da estimativa do conteúdo de carne magra da carcaça.

Tabela 6 - Equações de predição para estimar o peso (PCTT) e a porcentagem (RCTT) da porção comestível do corte traseiro a partir de medidas obtidas na carcaça

Table 6 - Equations of prediction to estimate for product hindquarter weigh (WRTH) and percent (RRPH) from carcass measures

\begin{tabular}{|c|c|c|c|c|c|c|c|c|c|}
\hline Variável e equação $^{1}$ & $\mathrm{R}^{2}$ & RMSE & Cp & Intercepto & EGSC (mm) & $\operatorname{AOLC}\left(\mathrm{cm}^{2}\right)$ & ACAB & CONF & PCQ (kg) \\
\hline Variable and equation & & RMSE & $C p$ & Intercept & CFAT (mm) & $C M L A\left(\mathrm{~cm}^{2}\right)$ & FAT & CONF & $H C W(\mathrm{~kg})$ \\
\hline
\end{tabular}

RCCT $(R R P H)$

\begin{tabular}{|c|c|c|c|c|c|c|c|c|c|}
\hline 5 & 0,66 & 0,99 & 4,29 & 83,16 & - & 0,01 & - & - & $-0,02$ \\
\hline 6 & 0,66 & 0,99 & 5,49 & 84,13 & - & 0,09 & $-0,01$ & - & $-0,02$ \\
\hline 5 & 0,66 & 0,10 & 6,25 & 83,08 & 0,01 & 0,10 & - & - & $-0,02$ \\
\hline 7 & 0,67 & 0,10 & 8,00 & 84,44 & 0,05 & 0,09 & $-0,19$ & $-0,09$ & $-0,02$ \\
\hline \multicolumn{10}{|c|}{ PCCT (WRPH) } \\
\hline 8 & 0,89 & 0,90 & 5,45 & $-0,86$ & - & 0,07 & 0,32 & - & 0,14 \\
\hline 9 & 0,89 & 0,91 & 8,26 & $-0,26$ & 0,01 & 0,06 & - & - & 0,14 \\
\hline 10 & 0,89 & 0,89 & 4,00 & 0,13 & - & 0,06 & 0,32 & $-0,15$ & 0,14 \\
\hline 11 & 0,89 & 0,89 & 6,00 & $-0,15$ & $-0,02$ & 0,06 & 0,40 & $-0,13$ & 0,14 \\
\hline
\end{tabular}

\footnotetext{
1 Ver Tabela 1 para descrição dos acrônimos.
}

1 See Table 1 for description of acronyms. 
A inclusão da AOLC elevou a explicação para 63\%, com a redução nos valores do Cp para 10 e RMSE para 1.03\%. Excetuando as covariáveis, a AOLC é a variável mais intensamente correlacionada $(\mathrm{r}=0,35, \mathrm{P}=0,0003)$ à RCTT . Portanto, foi a primeira variável a entrar no procedimento de seleção de variáveis Stepwise. A AOLC explicou, sozinha, $12,49 \%$ da porcentagem da porção comestível do corte traseiro, $1 \%$ a mais que a AOLUS. Cole et al. (1960) relataram coeficiente de correlação entre a AOLC e a porcentagem de carne magra da carcaça de 0,28 ao avaliarem mesma raça em mesmo período, similar às condições deste estudo.

No desenvolvimento do modelo completo para predição do RCTT considerando as características de carcaça (Tabela 5), verificou-se que o modelo deveria incluir pelo menos as variáveis AOLC e PCQ e as covariáveis \% OSSO e \% ATT, importantes na construção do modelo. Considerando que a \% OSSO e, principalmente, a \% ATT não são intensamente correlacionados ao PCQ, nesse caso, a inclusão do PCQ no modelo não eliminou a influência das covariáveis. O modelo selecionado foi com duas variáveis preditoras (AOLC e PCQ, nesta ordem de inclusão).

Os modelos de predição selecionados para o RCTT, a partir de medidas na carcaça podem dificultar sua aplicação prática, pois as covariáveis explicaram a maior parte da variação desta característica (Tabela 6). Portanto, seria necessário desossar as carcaças, pesar as aparas dos cortes e os ossos do corte do traseiro. Uma alternativa viável seria predizer a porcentagem da porção comestível do traseiro em porcentagem de aparas e ossos constante. Esse procedimento, segundo Herring et al. (1994), eliminaria o uso de proporções e, matematicamente, simplificaria os processos de predição, auxiliando no desenvolvimento das Diferenças Esperadas nas Progênies para estas características.

O rendimento da porção comestível ideal, em um peso de traseiro específico, poderia ser estabelecido e utilizado como parte de um índice. Entretanto, deveria ser estabelecida uma janela de aceitabilidade para os pesos de carcaças e dos cortes traseiros. Outro ponto importante para as equações obtidas no animal vivo e a comercialização dos animais para o abate. Com a estimativa do peso e da porcentagem da porção comestível a priori, é possível adotar um modelo de remuneração baseado no mérito individual do animal e não mais na média da população dos animais abatidos, como ocorre no atual sistema de produção.

\section{Conclusões}

As equações de predição obtidas a partir de modelos, tanto do animal vivo como de carcaça, apresentam alta força preditiva e são suficientemente acuradas para serem utili- zadas na estimativa do peso da porção comestível do corte traseiro da carcaça. As equações de predição obtidas a partir de modelos no animal vivo não apresentaram alto valor preditivo para estimar a porcentagem da porção comestível do corte traseiro da carcaça, no entanto, foram precisas para serem utilizadas em índices de seleção. A técnica de ultra-sonografia em tempo real consiste em ferramenta útil na estimativa da composição corporal em animais de abate.

\section{Literatura Citada}

ANIMAL ULTRASOUND SERVICE - AUS (AUSKey4W). A complete package for image and data analysis designed for use on an IBM/AT or compatible personal computer. Ithaca: Yujun Liu, Animal Ultrasound Services, 1994. 151p.

ABRAHAM, H.C.; CARPENTER, Z.L.; KING G.T. et al. Relationships of carcass weight, conformation and carcass measurements and their use in predicting beef carcass cutability. Journal of Animal Science, v.27, n.3, p.604-610, 1968.

BUSCH, D.A.; DINKEL, C.A.; SCHAFER, D.E. et al. Predicting edible portion of beef carcasses from rib separation data. Journal of Animal Science, v.27, n.2, p.351-354, 1968.

COLE, J.W.; ORME, L.E.; KINCAID, C.M. Relationship of loin eye area, separable lean of various beef cuts and carcass measurements to total carcass lean in beef. Journal of Animal Science, v.21, n.2, p.355, 1960.

CROSS, H.R.; CARPENTER, Z.L.; SMITH, G.C. Equations for estimating boneless retail cut yields from beef carcasses. Journal of Animal Science, v.37, n.6, p.1267-1272, 1973.

CROUSE, J.D.; DIKEMAN, M.E.; KOCH, R.M. et al. Evaluation of traits in the USDA yield grade equation for predicting beef carcass cutability in breed groups differing in growth and fattening characteristics. Journal of Animal Science, v.41, n.3, p.548-553, 1975.

CROUSE, J.D.; DIKEMAN, M.E. Determinates of retail product of carcass beef. Journal of Animal Science, v.42, n.3, p.584591,1976

CUTHBERTSON, A. Carcass evaluation of cattle, sheep and pigs. World Review of Nutrition and Dietetics, v.28, n.2, p.210235, 1978

GREINER, S.P.; ROUSE, G.H.; WILSON, D.E. et al. Prediction of retail product weight and percentage using ultrasound and carcass measurements in beef cattle. Journal of Animal Science, v.81, n.7, p.1736-1742, 2003.

HAMMOND, J. Growth and development of mutton qualities in sheep. Edimburgh: Oliver and Boyd, 1932. 595p.

HAMLIN, K.E.; GREEN, R.D.; PERKINS, T.L. et al. Real-time ultrasonic measurement of fat thickness and Longissimus muscle area: I. Description of age and weight effects. Journal of Animal Science, v.73, n.6, p.1713-1724, 1995.

HAMLIN, K.E.; GREEN, R.D.; CUNDIFF, L.V. et al. Real-Time ultrasonic measurement of fat thickness and longissimus muscle area: II. Relationship between real-time ultrasound measures and carcass retail yield. Journal of Animal Science, v.73, n.6, p.1725-1734, 1995a.

HERRING, W.; WILLIAMS, S.E.; BERTRAND, J.K. et al. Comparison of live and carcass equations predicting percentage of cutability, retail product weight and trimmable fat in beef cattle. Journal of Animal Science, v.72, n.5, p.1107-1118, 1994.

KEMPSTER, A.J.; CUTHBERTSOn, A.; JONES, D.W. Bone weight distribution in steer carcasses of different breeds and crosses, and prediction of carcass bone content from the bone content of joints. Journal of Agricultural Science, v.89, n.3, p.675-682, 1977. 
KEMPSTER, A.J.; CUTHBERTSON, A; HARRINGTON, G. Carcass evaluation in livestock breeding, production, and marketing. Great Britain: Granada Publishing, 1982. 306p.

MAY, S.G.; MIES, W. L.; EDWARDS, J.W. et al. Using live estimates and ultrasound measurements to predict beef carcass cutability. Journal of Animal Science, v.78, n.5, p.1255-1261, 2000.

MacNEIL, M.D. Choice of a predicting equation and the use of the selected equation in subsequent experimentation. Journal of Animal Science, v.57, n.5, p.1328-1336, 1983.

McKIERNAN, W.A.; WILKINS, J.F.; BARWICK, S.A. et al. CRC ' regional combinations' project- effects of genetics and growth paths on beef production and meat quality: experimental design, methods and measurements. Australian Journal of Experimental Agriculture, v.45, n.7, p.959-969, 2005.

MULLER, L. Normas para avaliação de carcaças e concurso de carcaças de novilhos. Santa Maria: Universidade Federal de Santa Maria, 1980. 31p.

Neter, J.; WASSERman, W.; KUTner, M.H. Applied linear statistical models: regression, analysis of variance, and experimental designs. 3.ed. Boston: Irwin, 1990. 1181p.

PERKINS, T.L. The use of real-time, linear-array ultrasound techniques to predict final carcass composition in beef cattle. Lubbock: Texas Tech University, 1992. 187p. Thesis (Ph. D. in Animal Science, Breeding and Genetics) - Texas Tech University, 1992.

REALINI, C.E.; WILLIAMS, R.E.; PRINGLE, T.D. et al. Gluteus medius and rump fat depths as additional live animal ultrasound measurements for predicting retail product and trimmable fat in beef carcasses. Journal of Animal Science, v.79, n.6, p.1378-1385, 2001.

STATISTICAL ANALYSIS SYSTEM- SAS. User's guide. version 8.2. Cary: 2001. 1686p.

SHAHIM, K.A.; BERG, R.T. Influence of bone growth on muscle growth and bone-muscle relationships in double-muscled and normal cattle. Animal Production, v.44, p.219-225, 1987.
STEWART, D.M. The role of tension in muscle growth. In: GOSS, R.J. (Ed.) Regulation of organ and tissue growth. New York: Academic Press, 1972. p.77-100.

TAIT, J.R.; ROUSE, G.H.; WILSON, D.E. et al. [2000]. Prediction of lean in the round using ultrasound measures. Iowa State University, Beef Research Report. A.S. Leaflet R1733. Disponível em: http://www.iowabeefcenter.org/pages/ansci/ beefreports/asl1733.pdf. Accesso em: 8/4/2005.

TAIT, J.R.; ROUSE, G.H.; WILSON, D.E. Comparison of ultrasound and carcass measures to predict percentage of beef retail product from four primal cuts: final report. Ames: Iowa State University, 2003. 3p. (Beef Research Report).

TAIT, J.R.; WILSON, D.E.; ROUSE, G.H. Prediction of retail product and trimmable fat yields from the four primal cuts in beef cattle using ultrasound or carcass data. Journal of Animal Science, v.83, n.6, p.1353-1360, 2005.

WALLACE, M.A; STOUFFER, J.R.; WESTERVELT, R.G. Relationships beef yield from ultrasonic and carcass measurements with retail yield in beef cattle. Livestock Production Science, v.4, n.2, p.153-164, 1977.

WILLIAMS, R.E.; BERTRAND, J.K.; WILLIAMS, S.E. et al. Biceps femoris and rump fat as additional ultrasound measurements for predicting retail product and trimmable fat in beef carcasses. Journal of Animal Science, v.75, n.1, p.7-13, 1997.

WOLCOTT, J.M.; THOMPSON, D.; PERRY, D. The prediction of retail beef yield from real-time ultrasound measurements on live animals at three stages through growout and finishing. Australian Journal of Experimental Agriculture, v.41, n.7, p.1005-1011, 2001. 